\title{
An advanced blind-tube monitoring instrument to improve the characterization of subsurface radioactive plumes
}

\author{
Soraia Elísio ${ }^{1}$, Malcolm J. Joyce ${ }^{1}$, James Graham ${ }^{2}$, Barrie Greenhalgh ${ }^{3}$ \\ ${ }^{1}$ Department of Engineering, Lancaster University, United Kingdom \\ ${ }^{2}$ Central Laboratory, National Nuclear Laboratory Ltd., United Kingdom \\ ${ }^{3}$ Radiometric Systems Group, Sellafield Ltd., United Kingdom \\ s.elisio@lancaster.ac.uk
}

\begin{abstract}
This research presents a design of a resilient blind-tube radiometric logging probe, which satisfies key, operational site constraints, for direct in-situ characterization of subsurface radioactivity, particularly caesium-137 and strontium-90. The probe comprises a commercially-available $\emptyset 10 \mathrm{~mm} \times 9.5 \mathrm{~mm} \mathrm{CeBr}_{3}$ scintillator detector attached to a compact digitizer unit, in a resistant and waterproof housing. The probe is designed to be lowered down into metallic $\varnothing 75 \mathrm{~mm}$ blind-tubes by means of a winch system.
\end{abstract}

Keywords — blind-tube logging, $\mathrm{CeBr}_{3}$ scintillator, gamma-ray spectroscopy, decommissioning

\section{INTRODUCTION}

$\mathrm{T}$ HE wet interim storage of nuclear waste in ponds and silos is a common approach at nuclear licensed sites. However, some of these legacy facilities were not designed to modern building standards, and some cases leakage of aqueous radioactive into the subsurface has occurred, dispersing downward and outward in the ground and contaminating the soil and sediment along its path. If unmitigated, then these plumes pose a potential risk to groundwater, surface water receptors and subsequently public health and the environment. nearby streams, presenting an elevated risk to public health and the environment.

Licensed sites have specific regulations associated with the protection and assessment of the soil and groundwater associated with such facilities. Monitoring programs are often focused on the detection and characterization of sources of contamination, and on the evaluation of temporal changes through surveillance measurements at target locations. Additionally, the spread of contamination can be reduced via emplacement of engineered barriers, or remediation schemes ensuring the preservation of the baseline quality of the soil [1] and groundwater [2-5].

The facility on which this research is focused is the Magnox Swarf Storage Silos (MSSS). It was built in the 1960s in Sellafield (UK) and expanded three times during 1970s and 1980s. There are a total of 22 individual vertical concrete silos installed partially below ground level. The aging compartments contain a mixture of intermediate level solid waste underwater from Magnox's reprocessing for approximately forty years. In addition, fuel corrosion sludge residues and swarf stock contain large amounts of various beta gamma residues. The MSSS is a legacy facility currently assessed as the highest risk nuclear facility in the Nuclear Decommissioning Authority estates. MSSS was not built to modern standards and the risk continues to increase significantly with time as the building structure ages. SL has commenced a programme of work to the retrieve solid waste from MSSS and transport it for storage in a more modern storage facility [6,7]. It is known that liquor from the MSSS silos have leaked into the ground contaminated the land below the silo, in the 1970s [6,7], and more recently [7, 8] and this contamination is the focus of routine radiological surveillance [9-10].

The current regulations follow the contamination land best practice risk management system to assess the impact of release, or remobilization, of radioactive contaminants. This involves both a routine groundwater sampling programme and more focused site characterization activities involving borehole drilling and associated soil sampling. The samples are processed and analyzed in the laboratory [11]. Sampling and laboratory-based radiological measurements are well established and provide accurate identification and quantification of gamma-emitting, and most prominent hard-tomeasure alpha/beta-emitting radionuclides. However, these approaches are limited by: the datasets being discrete; the practice is expensive, laborious, and time-consuming; the treatment steps generate secondary wastes, and there can be considerable radiological risk to workers.

Alternative methods, such as down-hole radiometric logging systems, have also been applied as the optimal method to characterize underground radioactivity. These solve some practical limitations imposed by the traditional method, as it provides continuous, real-time assessment of vertical soil profiles, is less intrusive approach and allows multiple measurements to investigate trends of contamination distribution over the time. Besides this it also enables investigation of a volume of ground many times larger than the borehole size. Further, if a grid of boreholes is available, this mode of deployment can be extended, and a three-dimensional model of the subsurface could be derived from the data.

Borehole logging simply consists of a continuous recording of a certain physical property as a function of depth, inside boreholes (or drill holes). The recording device (logging probe) comprises a sensing system, and its associated electronic 
circuity, in a resistant and waterproof case. The logging probe is connected to a cable and lowered downhole by means of a winch system, and information on the probe position in the borehole is provided by a depth encoder. The logging cable provides data transfer, power supply, and mechanical support to lower and raise the probe [12].

In the context of this research, we are interested in direct assessment of radioactive contamination in the ground, this is, to locate, identify and quantify radionuclides. Therefore, a borehole logging probe comprising ionizing radiation detectors as sensor system is highly desirable. Radiometric logging originally evolved from geoscience applications for measure natural gamma-ray radioactivity from rocks in and around oilbearing formations for example [13-15]. It has also been applied to aid numerous underground assessments at nuclear waste disposal sites, such as the Oak Ridge site (Tennessee, US), the Maxey Flats site (Kentucky, US) [13,15], and the Hanford site (Washington, USA) [17].

The limitations of borehole approach are mostly dominated by the need of construction of ground assets, and the intrinsic limitations of the radiometric detectors. The obstruction by belowground assets on site and the limiting penetration power of radioactivity in matter may limit the ease with which horizontally-resolved measurements are obtained, as well. Moreover, there is a significant challenge to detect principally alpha-/beta-emitting radionuclides given the low penetration power characteristic of such radiations, despite their frequent association with nuclides of significant radiotoxicity and therefore concern.

The challenge monitoring of the ground beneath the MSSS legacy facility is compounded by operational infrastructure and deployment constraints due to the complexity and congestion of the site $[9,10]$. One existing in-ground monitoring asset local to MSSS building are an array of carbon steel blind-tubes, of around $10 \mathrm{~m}$ depth, and inner diameter of $75 \mathrm{~mm}$. The radioactivity fingerprint in the soil monitored by the blind-tube array is believed to be dominated by caesium-137 with strontium-90 and non-isotopic specific dose measurements in some of the blind-tubes reach $1 \mathrm{~Gy} / \mathrm{h}$. These dose rates represent a significant limitation in the choice of detectors which may be deployed and their long-term functionality.

The radiometric logging probes used in such scenarios, typically comprise gamma-ray detectors such as gas-filled detector Geiger-Muller (G-M) tubes [17], sodium-iodide (NaI:Tl) scintillators [13-18] and portable high-purity germanium (HPGe) detectors [17-19].

GM tubes have the advantage of being robust, simple, and inexpensive detectors; however, they do not discriminate between different types of radiation, nor radiation energies. As a result, GM tubes are only applied as for basic survey of gross dose rate. Nevertheless, depending upon the specific application, GM tubes perform adequate assessment of radiation levels and trends or changes over time.
NaI:Tl scintillation crystals are the most extensively used sensing material for gamma spectroscopy applications. Sodium iodide is cheap, highly efficient, available in varying sizes and geometries, and very well characterized in the literature. The resolution is sufficient to speciate dominant and well-separated characteristic peaks of some radionuclides, allowing relatively fast surveys at depth. However, the relatively low-resolution characteristic of NaI:Tl might lead to inaccurate analysis when used in some applications. For scenarios comprising a mixture of radioactive materials, shielded and with a high background, a device with higher energy resolution is essential to resolve and clearly distinguish gamma lines in the associated complex spectra. Another limitation of NaI:Tl detectors is their slow scintillation decay time constants. Thus, when deployed in a high radiation level environment the signals tend to pile up. Moreover, NaI:Tl crystals are also susceptible to performance degradation from radiation damage for dose levels above $1 \mathrm{~Gy}$ [20].

HPGe semiconductor detectors hold excellent energy resolution, which provide accurate analysis of distinct gamma energies and thus outstanding identification of radionuclides in a wide range of scenarios. The limitation of HPGe detectors is their high cost and the requirement to operate at cryogenic temperatures. Recent advances in HPGe technology have resulted in modules with reduced size and complexity, with electromechanical cooling, and although still expensive are promising in terms of their potential deployment in small spaces.

The selection of an optimal technology for this application is a crucial step to achieve the purpose of this research, with an alternative detection material desirable to ensure quality in underground gamma-ray spectrometry. This will fill the gap between low-resolution scintillators and expensive semiconductor detectors. So, on this basis, the new radiometric logging probe system shall:

- Improve data gathering in existing metallic assets,

- Be operational at high count rates

- Have high intrinsic radiation hardness,

- High energy resolution, and

- Be compatible to be left in harsh environmental conditions.

The proposed solution consists of a scintillator gamma detection system. Among the many available scintillating materials (see Table 1), a small lanthanide halide cerium bromide $\left(\mathrm{CeBr}_{3}\right)$ crystal was chosen as an optimal compromise with good key characteristics for this application. It has good gamma-ray detection efficiency (due to a high effective atomic mass), good energy resolution, fast decay time and high radiation hardness. Moreover, its reduced intrinsic activity also offers an advantage over $\mathrm{LaBr}_{3}$ for accurate measurements of low-intensity gamma rays. 
TABLE I

AVAILABLE CANDIDATE SCINTILLATOR MATERIALS AND KEY PROPERTIES [20-29]

\begin{tabular}{lcccccc}
\hline \hline Key Parameters & $\mathrm{NaI}: \mathrm{Tl}$ & $\mathrm{LaBr}_{3}$ & $\mathrm{CeBr}_{3}$ & $\mathrm{CLYC}: \mathrm{Ce}$ & $\mathrm{CLLB}: \mathrm{Ce}$ & GAGG:Ce \\
\hline $\begin{array}{l}\text { Detection efficiency } \\
\text { Zeff; } \rho\end{array}$ & $51 ; 3.7$ & $47 ; 5.1$ & $46 ; 5.2$ & $54 ; 3.3$ & $47 ; 4.1$ & $54 ; 6.7$ \\
$\begin{array}{l}\text { Energy resolution } \\
\text { FWHM @ 662 keV }\end{array}$ & $6.5-10 \%$ & $2.5-3 \%$ & $3.2-4 \%$ & $4.5-5 \%$ & $2.9-3.5 \%$ & $4.5-4.8 \%$ \\
$\begin{array}{l}\text { Radiation hardness } \\
\text { Dose [Gy] @ Gamma-ray }\end{array}$ & 1 & $10^{5}$ & $10^{5}$ & - & - & $10^{3}$ \\
$\begin{array}{l}\text { High count-rate capability } \\
\tau \text { [ns] }\end{array}$ & 230 & 16 & 17 & $\begin{array}{c}50 \\
1000\end{array}$ & 120 & 800 \\
Hygroscopic & Yes & Yes & Yes & Yes & Yes & No \\
\hline \hline
\end{tabular}

Drawing upon strands of research into the desirable properties of a gamma-ray optimal detector, this study also aims to improve in-ground radiological risk assessment of long-lived gamma-emitting fission products, such as caesium-137, and energetic beta emitters, such as strontium-90 (derived via detection of the associated bremsstrahlung radiation).

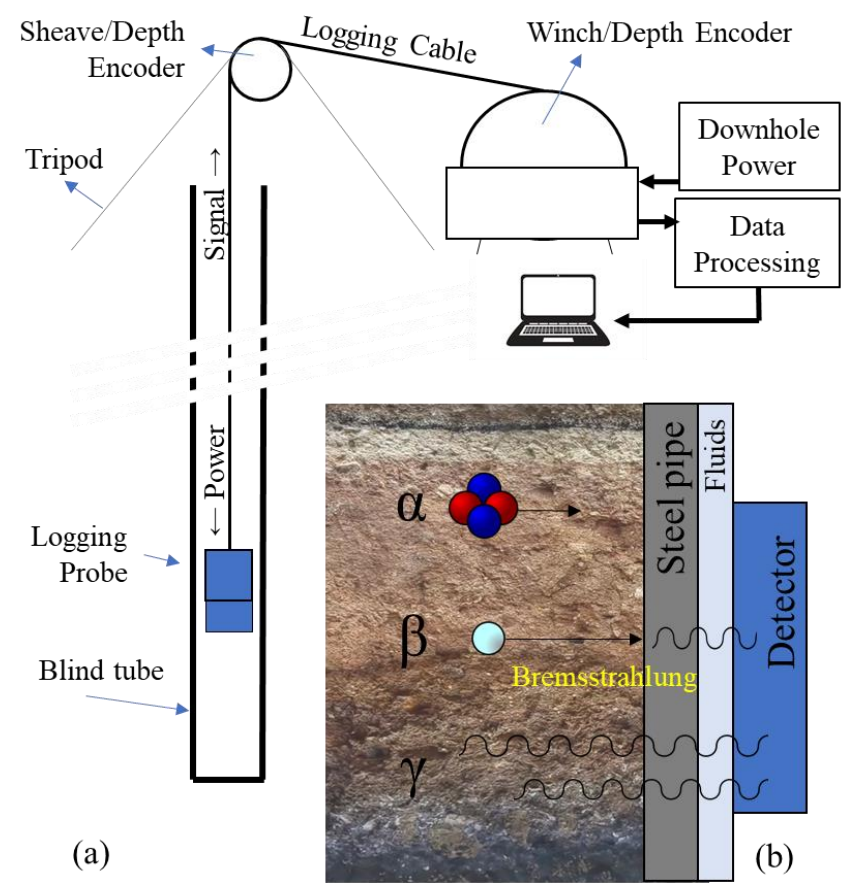

Figure 1 - A schematic diagram of a (a) blind-tube radiation sensing and downhole deployment system to (b) detect gamma and energetic beta emitting radionuclides (via produced bremsstrahlung photons) present in the surrounding soil formation (but close to the blind-tube). Alpha and beta lowenergy emitting radionuclides are out of the scope of detection.

\section{THE BLIND-TUBE RADIOMETRIC PROBE}

The proposed blind-tube radiometric logging probe is comprised of a gamma-ray spectrometer. The sensing material is a $\varnothing 10 \mathrm{~mm} \times 9.5 \mathrm{~mm}$ cerium bromide $\left(\mathrm{CeBr}_{3}\right)$ scintillation crystal which is coupled to a silicon photodiode photomultiplier (PM6660-SiPM), and a preamplifier, in a single unit (VS-140220) commercialized by Scionix (Netherlands) (figure $2 a$ ). This selection satisfies the key characteristics for the application highlighted above. It has good gamma-ray detection efficiency
$\left(Z_{\text {eff }}=46, \rho=5.2 \mathrm{~g} / \mathrm{cm}^{3}\right)$, energy resolution $(3.2-4 \% @ 662 \mathrm{keV})$, high count-rate capability (decay time $=17 \mathrm{~ns}$ ) and radiation hardness $\left(10^{5} \mathrm{~Gy}\right)$ [22-24]. Note that the SiPM device has practical advantages over traditional photomultiplier tubes, such as low voltage operation, insensitivity to magnetic fields, and superior roughness.

The signal processing chain is accomplished via a digital processor module placed downhole in the probe. The main advantage of digitizing the signal at the probe is that the digital signal can convey information over long distances with less noise, distortion, and environment interference, in contrast with analogue data transmission. Moreover, digitizers are more userfriendly and flexible, and available in a compact unit compatible with the dimensions of typical blind-tubes. Furthermore, processing operations can be altered, and parameters optimized using programmable software to suit a specific application. The proposed digitizer is the compact, stand-alone digital Multi-Channel Analyser (MCA) TopazSiPM module commercialized by BrightSpec NV (figure $2 \mathrm{~b}$ ). Note that it is designed to process pulses produced by the silicon photodiode photomultiplier (SiPM) detector, and that it also provides the necessary low-ripple and low-noise $5 \mathrm{~V}$ voltage supply for the SiPM preamplifier to operate. Therefore, a highvoltage power supply unit is not needed, and the probe can be easily powered from the PC via USB, or Ethernet (PoE) cable. Another important consideration is the applicability of the $\mathrm{CeBr}_{3}+\mathrm{MCA}$ system to high radiation environment (figure 3).

(a)

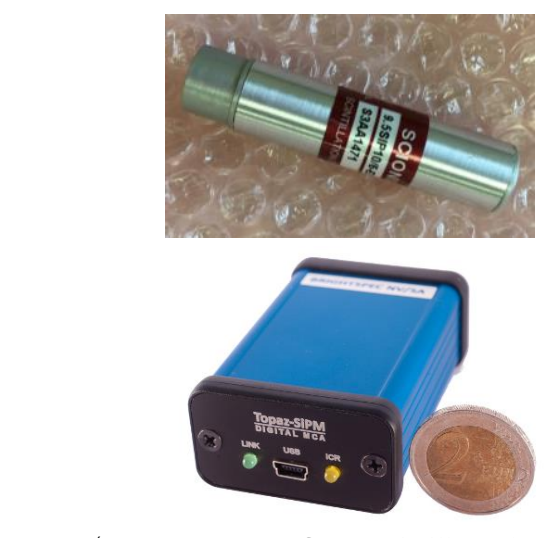

(b)

Figure 2 - (a) Ø10 $\mathrm{mm} \times 9.5 \mathrm{~mm} \mathrm{CeBr}$ scintillator detector, with PM6660SiPM and built-in preamplifier commercialised by Scionix. (b) Topaz-SiPM digital MCA module commercialised by BrightSpec NV. 


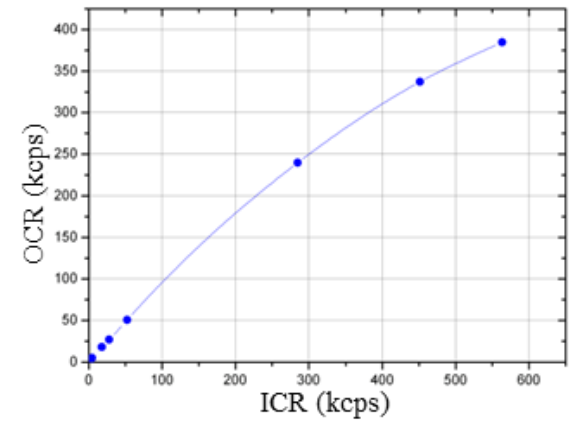

Figure 3 - High count rate capability of system $\varnothing 10 \mathrm{~mm}$ x $10 \mathrm{~mm} \mathrm{CeBr}{ }_{3}+$ SiPM with Topaz-SiPM MCA, applying short shaping times ( $\mathrm{RT}=0.8, \mathrm{FT}=1$ ). The radiation source sample was a technetium-99 bottle (figure given by the manufacturer).

\section{THE BLIND-TUBE TEST PIT}

Gamma-ray spectrometers must be calibrated to perform quantitative measurements of the emissions from radionuclides, and such calibrations are specific to the sample geometry of the field measurement. In borehole logging measurements, the probe is virtually surrounded by the source, being effectively in a $4 \pi$ geometry, excluding only a small solid angle above and below it. Traditionally, nuclear probes for geophysical application are calibrated in primary borehole models (or test pits) containing known and uniform concentration of potassium (K model), uranium (U model), or thorium ( $\mathrm{T}$ model) minerals [14].

Limitations fall to the extent of what is possible and practical to mimic the characteristics of the ground, such as density and composition. The detector response function is defined for a particular geometry, density, and material composition, and correction factors are needed for other conditions [14,30]. Monte Carlo simulations provide an alternative source from which to obtain the detector response functions for a single radionuclide, or decay series, without interference from other radionuclides, and to investigate the effects of heterogeneities in the formation, for example. However, models must be validated with experimental data [30,31].

Figure 4 shows the design and construction of a blind-tube test bed that replicates as close as practical the existing inground blind-tubes, such as solid angle coverage of the detector and blind-tube characteristics. Moreover, sand is used as the ground content to facilitate simulations.
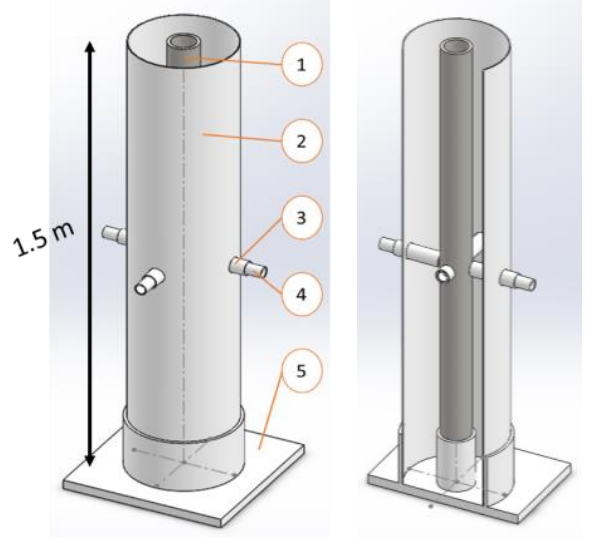

(b)
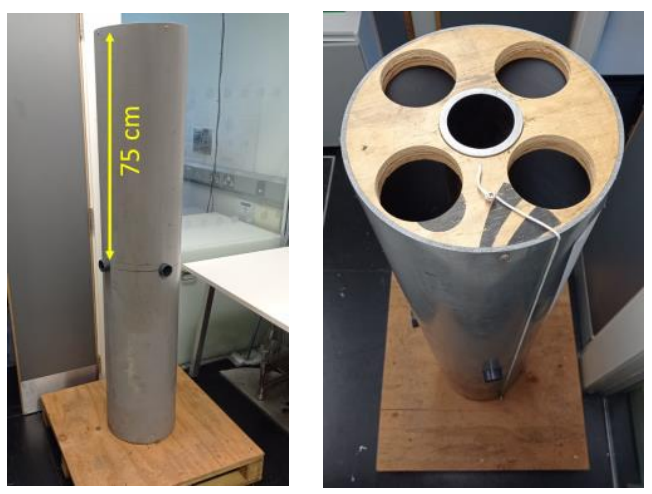

Figure 4 - (a) CAD design of the blind-tube test bed assembled using Solidworks. It includes (1) a carbon steel tube with inner diameter of $75 \mathrm{~mm}$, wall-thickness of $9.5 \mathrm{~mm}$, and cut to a length of $1.5 \mathrm{~m}$, (2) a plastic sand retaining tube with outer diameter of about $320 \mathrm{~mm}$, wall-thickness of $5 \mathrm{~mm}$, and cut to a length of $1.5 \mathrm{~m},(3)$ four plastic 'void' tubes placed horizontally at $75 \mathrm{~cm}$ from the top, (4) four plastic tubes to hold Ø $25 \mathrm{~mm}$ disk point sources, and (5) the base support. (b) Picture of the current configuration.

\section{DETECTOR PERFORMANCE}

This section presents preliminary desk studies under laboratory conditions to determinate the gamma-ray spectral performance and the angular dependence of response of a cerium bromide scintillator detector, and to test the hypothesis of detecting indirectly beta-emitting nuclide strontium-90, via the bremsstrahlung radiation produced in the materials surrounding its location.

\section{A. Experimental setup}

The detection setup comprises a $\varnothing 10 \times 10 \mathrm{~mm} \mathrm{CeBr}_{3}$ scintillation crystal, coupled to a photomultiplier Hamamatsu R9880-110, and an in-built HV-supply and voltage divider, commercialized by Scionix (unit VS-0087-50). The detector readout was connected to digitizer MCA4 commercialised by FAST ComTec (sourced from JCS Ltd., UK). Data were transferred to a computer via USB 2.0 interface and spectra analysed using the MPANT-MCA4 software. The gamma- and beta-emitting disk sources used in this campaign were caesium-137 (304 $\mathrm{kBq})$, europium-152 (370 kBq) and strontium-90 (370 kBq). Measurement times were long enough to achieve good statistical precision for peak evaluation. Figure 5 presents the detector system applied in this campaign of measurements.

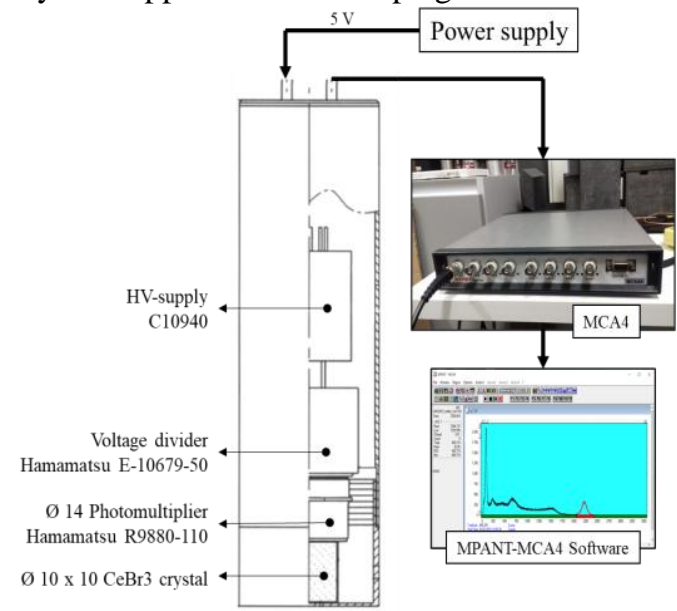

Figure 5 - Simple schematic of detector assembly and experimental detection system used for the preliminary desk studies. 


\section{B. Results and discussion}

To study the gamma spectroscopy performance of the detector system an Cs-137 and Eu-152 gamma-ray point sources were placed perpendicularly to the central axis of the detector, centered to its sensing volume (figure 6). This geometric configuration is equivalent to a situation in which the detector is positioned vertically in a hole with radioactive material present on the side.

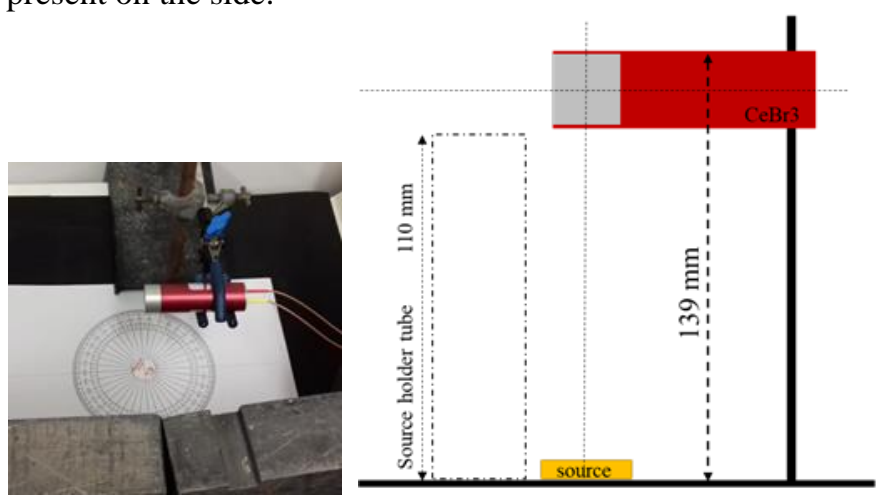

Figure 6 - Experimental setup applied to study the detector gamma-ray spectral performance.

TABLE II

GAMMA-RAY ENERGIES AND EMISSION PROBABILITIES FOR THE RADIONUCLIDES USED IN THE EXPERIMENT [32]

\begin{tabular}{lll}
\hline \hline Nuclide & Energy (keV) & Emission probability \\
\hline Cs-137 & $661.657(3)$ & $0.8499(20)$ \\
Eu-152 & $121.7817(3)$ & $0.2841(13)$ \\
& $244.6974(8)$ & $0.0755(4)$ \\
& $344.2785(12)$ & $0.2658(12)$ \\
& $411.1165(12)^{*}$ & $0.02237(10)$ \\
& $443.965(3)^{*}$ & $0.03125(14)$ \\
& $778.9045(24)$ & $0.1296(6)$ \\
& $867.38(3)$ & $0.04241(23)$ \\
& $964.072(18)$ & $0.1462(6)$ \\
& $1085.837(10)^{*}$ & $0.1013(6)$ \\
& $1089.737(5)^{*}$ & $0.01731(10)$ \\
& $1112.076(3)^{*}$ & $0.1340(6)$ \\
& $1212.948(11)^{*}$ & $0.01415(9)$ \\
& $1299.142(8)^{*}$ & $0.01632(9)$ \\
$1408.013(3)$ & $0.2085(9)$ \\
\hline \hline
\end{tabular}

* Not included in spectral analysis calculations.

Regions-of-interest (ROI) of each peak were set manually, and a single Gaussian peak fit with linear background was applied using the MPANT software. The respective centroidpeak and net-peak area values were extracted. Table 2 shows a list of the expected gamma-ray energies and emission probabilities for the radionuclides $\mathrm{Cs}-137$ and $\mathrm{Eu}-152$. Note the selected energies in the calibration procedure.

The gamma-ray spectra are shown in figure 7 , as well as the energy and peak width calibration curves. These results suggest a good detector response linearity in the energy range considered 122 to $1408 \mathrm{keV}$, with the most prominent nuclide peaks well distinguished from background. The detector energy resolution and the photopeak efficiency achieved at $662 \mathrm{keV}$ was $5.2 \%$ and $0.002 \%$ respectively. This resolution values is analogous to the result presented in [24], nevertheless higher than the value obtained by the manufacturer. Moreover, overlapping peaks can be distinguished by fitting anomalous peak shapes on the basis of inconsistent FWHM values, relative to the calibration curve. This might be useful to indicate the existence of multiple peaks in each region.

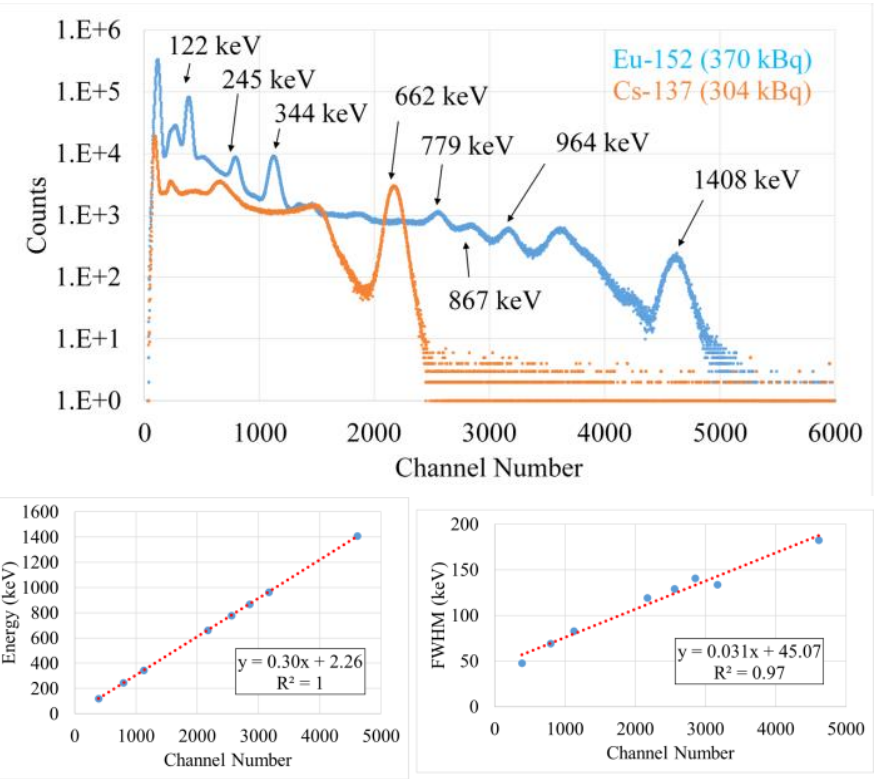

Figure $7-\varnothing 10 \times 10 \mathrm{~mm}^{2} \mathrm{CeBr}_{3}+$ digitizer MCA4 gamma-ray spectrum for Cs137 and Eu-152 isotropic point sources. Energy calibration curve and energy resolution response. Acquisition time of about $19 \mathrm{~h}$ and $25 \mathrm{~h}$ respectively.

The angular response of the detector to an isotropic point source was obtained. Measurements were performed placing the detector to a turntable with its sensing volume centered on the rotation axis and a Cs-137 gamma-ray source fixed along the axis of the cylindrical detector at $10 \mathrm{~cm}$ from it. Thus, the source testing path is a circle around the crystal center with radius of $\mathrm{D}=105 \pm 2 \mathrm{~mm}$ (figure 8 ).

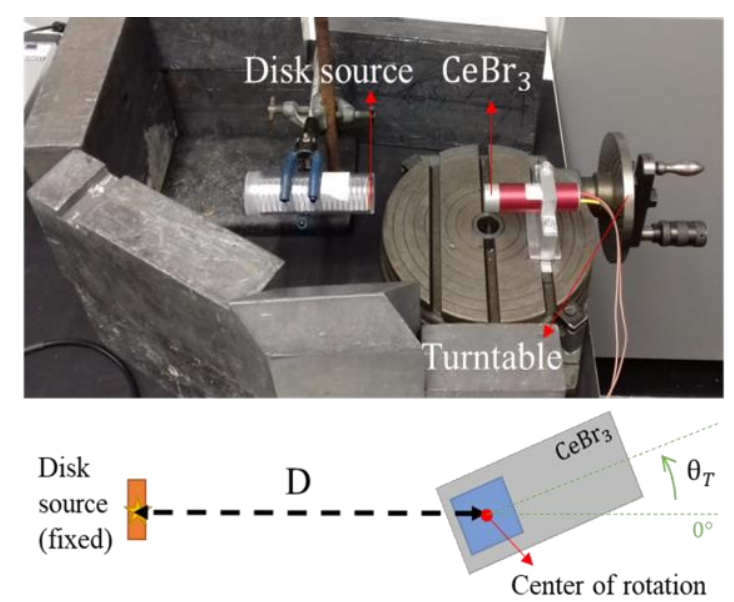

Figure 8 - Experimental setup applied to study the angular dependence of the detector.

Figure 9 shows the detector angular dependence curves for Cs-137 photopeak $662 \mathrm{keV}$ and $\mathrm{x}$-ray $32 \mathrm{keV}$ energies. No significant variation of response was observed over the angle of incidence, and despite the cylindrical shape, the detector may be considered independent of angular directions over the energy range of interest. 

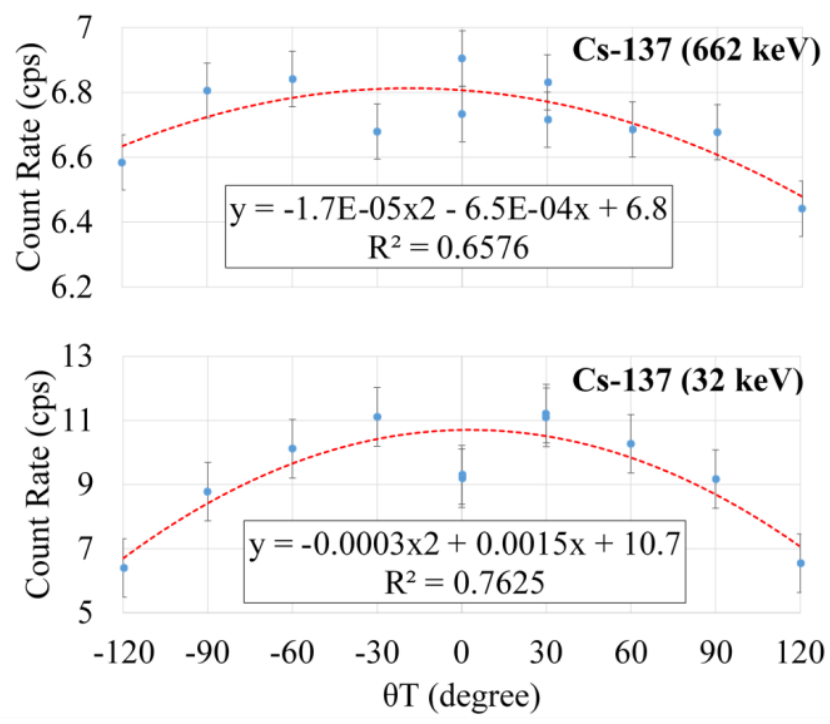

Figure 9 - Angular dependence of a $\varnothing 10 \times 10 \mathrm{~mm}^{2} \mathrm{CeBr}_{3}$ detector for Cs-137 photopeak $662 \mathrm{keV}$ and x-ray $32 \mathrm{keV}$ energies. Acquisition times of about $1 \mathrm{~h}$ each point

Another experiment was the test of the hypothesis of detection Sr-90 (hence Y-90) via produced bremsstrahlung radiation in the presence of gamma-ray emitting sources. The detector was placed centered in a $\varnothing 73 \times 88 \mathrm{~mm}$ PVC case and inserted in a carbon steel tube (figure 10). Here, Cs-137 and Sr90 disk sources were fixed to the outside of the metallic tube wall. Spectra were obtained for the situations where only Cs137 is present next to the wall, only Sr-90, or where both are presented (figure 11).
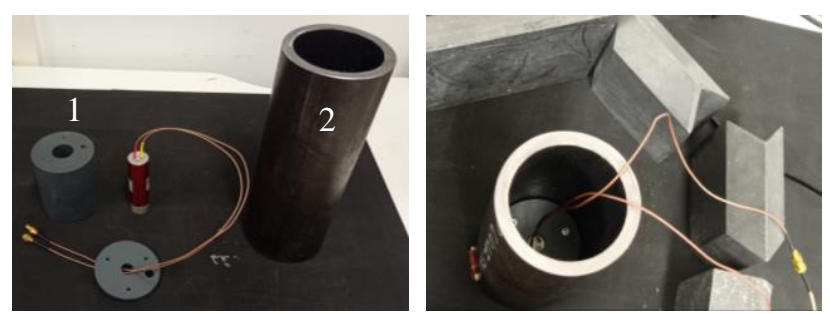

Figure 10 - Experimental setup applied to study the Sr-90 (via Bremsstrahlung) + Cs-137 assessment. (1) is PVC cylinder with outer size $\varnothing 73 \times 88 \mathrm{~mm}$, and a cavity to insert the detector centered. (2) is a $25 \mathrm{~cm}$ cut of the carbon steel tube presented in the blind-tube test pit section.
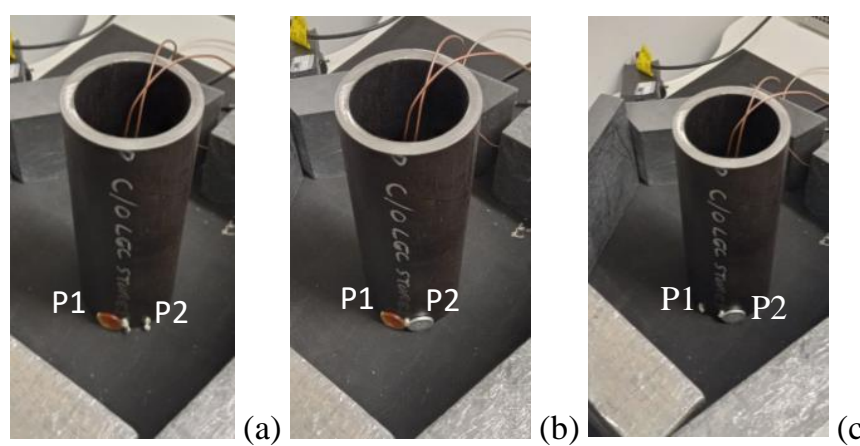

Figure 11 - (a) Cs-137 source placed at position P1. (b) Cs-137 source placed at position $\mathrm{P} 1+\mathrm{Sr}-90$ source placed at position $\mathrm{P} 2$. (c) Sr-90 source placed at position $\mathrm{P} 2$.

Figure 12 shows the detector's response for the three situations mentioned above. The results show that the detection of Sr-90 is feasible applying this method for the activity sources considered. Furthermore, the difference in shape of the Cs-137 and Cs-137+Sr-90 spectrum suggest the possibility of discrimination between the different radiation contributions.

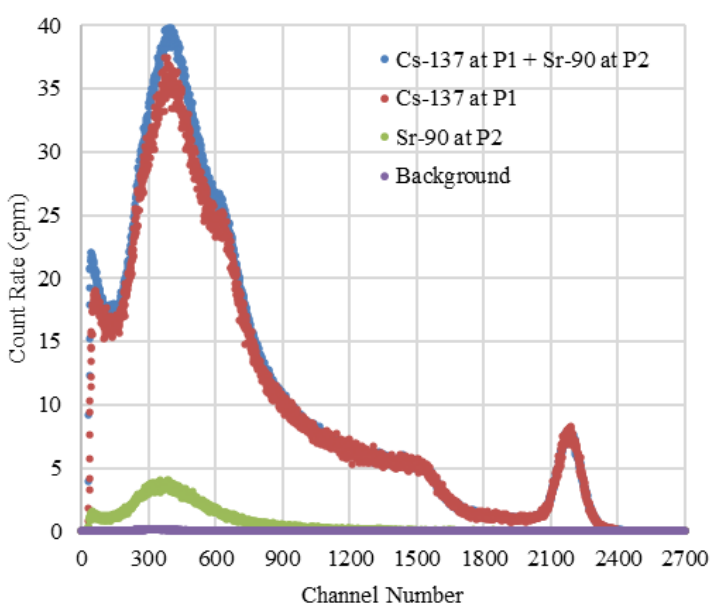

Figure 12 - Measured spectra of bremsstrahlung radiation and gamma-ray radiation of Sr-/Y-90 and Cs-137 respectively. The sources were placed in outer side of a $1 \mathrm{~cm}$ wall carbon steel tube.

\section{CONCLUSIONS AND FUTURE WORK}

This paper reports a design of a blind-tube monitoring instrument to improve the characterization of subsurface radioactive plumes beneath the MSSS facility at the Sellafield site (UK). Other prospective uses envisaged are to aid environmental and safety monitoring in and around reactor fuel processing plants, leak detection at interim storage sites for high level waste, and in other worldwide nuclear operating sites which are undergoing land remediation as part of decommissioning.

Overall, the unit comprises a $\varnothing 10 \times 9.5 \mathrm{~mm} \mathrm{CeBr}_{3}$ scintillation detector attached to a compact, stand-alone digitizer TopazSiPM module in a resistant and waterproof housing.

Preliminary experimental studies were carried out to study the gamma-ray spectral performance and angular dependence of a small $\emptyset 10 \times 10 \mathrm{~mm} \mathrm{CeBr}_{3}$ scintillation detector. Moreover, a design of a blind-tube test bed for efficiency calibration purposes is also included in this work. Additionally, the hypothesis of detecting $\mathrm{Sr}-90$ via bremsstrahlung radiation has also been assessed, including measurements of this isotope in the presence of Cs- 137 .

The detector features observed suggest that the $\varnothing 10 \times 10 \mathrm{~mm}$ $\mathrm{CeBr}_{3}$ detector is a promising solution for monitoring underground gamma-emitting radionuclides and high energetic beta-emitting radionuclides, addressing geometric requirements imposed by the existing blind-tubes on site.

Validation of the concept on the blind-tube test pit are underway, alongside with development of a Monte Carlo model in Geant4 software. As well as investigation of a method able to discriminate the contribution of bremsstrahlung photons from the Compton scattering photons at low energy and quantify this discrimination.

Long term future work involves testing of the spectroscopy performance of the system under high count rate conditions, as well as radiation hardness, as well as temperature and shock tests. Finally, the ultimate goal involves extension of a single 
detector to a blind-tube string network and create a 3dimensional picture of radiation present on the ground within the MSSS building in the Sellafield site.

\section{ACKNOWLEDGMENT}

This research is supported by NNL and Sellafield Ltd. (UK) via the EPSRC programme TRANSCEND (TRANSformative Science \& Engineering for Nuclear Decommissioning), code EP/S01019X/1. M. J. J. acknowledges the support of the Royal Society via a Wolfson Research Merit Award.

\section{REFERENCES}

[1] ISO, B. "Measurement of radioactivity in the environment - Soil," BS ISO, 18589, Part 2, 2017, Part 3, 2007, Part 5, 2009, Part 6, 2009.

[2] ISO, B. "Water quality - Sampling," BS ISO, 5667, Part 11, 2009, Part 22,2010 .

[3] ISO, B. "Geotechnical investigation, and testing - Sampling methods and groundwater measurements," BS ISO, 22475, Part 1, 2006.

[4] ISO, B. "Water quality - Determination of the activity concentration of radionuclides - Methods by high resolution gamma-ray spectrometry," BS ISO, 10703, 2007

[5] ISO, B. "Water quality - Strontium 90 and strontium 89 - Test methods using liquid scintillation counting or proportional counting," BS ISO, 13160, 2020.

[6] ONR, "Magnox Swarf Storage Silo (MSSS) - Risk and Hazard Reduction Programme Application for Agreement to Commence Installation and Inactive Commissioning of SEP2 and 1 Mobile Caves in MSSS, “ Office for Nuclear Regulation, 2016. [Online]. Available: https://www.onr.org.uk/pars/2015/sellafield-15-008.pdf. Accessed on: 14 July, 2021.

[7] ONR, "Magnox Swarf Storage Silo Retrievals Project Agreement to implement Phase 2 active commissioning trials of the MSSS compartment 10 Miscellaneous Beta Gamma Waste retrieval scheme," Office for Nuclear Regulation, 2019. [Online]. Available: https://www.onr.org.uk/pars/2019/sellafield-19-005.pdf. Accessed on: 14 July, 2021.

[8] ONR, "Quarterly Statement of Civil Incidents reported to ONR," Office for Nuclear Regulation, 2019. [Online]. Available: https://www.onr.org.uk/quarterly-stat/2019-4.htm. Accessed on: 14 July, 2021.

[9] Sellafield Ltd., "Delivering nuclear innovation - Characterisation and monitoring using in-ground assets," Game Changers, 2020. [Online]. Available: https://www.gamechangers.technology/static/u/Characterisation\%20and $\% 20$ Monitoring\%20Challenge\%20Statement.pdf. Accessed on: July 7, 2021.

[10] Sellafield Ltd., "Game Changers webinar - Characterisation and monitoring using in-ground assets," Game Changers, 2020. [Online]. Available: https://vimeo.com/458899566. Accessed on: July 7, 2021.

[11] Sellafield Ltd., "Groundwater monitoring at Sellafield - Annual data review," Report n. LQTD000758, 2016.

[12] Erdi-Krausz, G., Matolin, M., Minty, B., Nicolet, J. P., Reford, W. S., \& Schetselaar, E. M., "Guidelines for radioelement mapping using gamma ray spectrometry data," International Atomic Energy Agency (IAEA), 2003.

[13] Keys, W. S., "A practical guide to borehole geophysics in environmental investigations," CRC Press, 1996.

[14] Schubert, G., "Treatise on geophysics," Elsevier, 2015.

[15] Ellis, D. V., \& Singer, J. M., "Well logging for earth scientists", Vol. 692, Dordrecht: Springer, 2007.

[16] Keys, W. S., Eggers, D. E., \& Taylor, T. A., "Borehole geophysics as applied to the management of radioactive waste: site selection and monitoring," In Management of low-level radioactive waste, Volume II, 1979.

[17] Johnson, M. E., \& Field, J. G., "Hanford SX-Farm Leak Assessments," Report In RPP-ENV-39658, Rev. 0, Washington River Protection Solutions Richland, Washington, 2010.

[18] Keys, W. S., Senftle, F. E., \& Tanner, A. B., "Use of NaI(Tl) and germanium detectors for in situ X-ray spectral monitoring of boreholes at nuclear waste-disposal sites," No. 79-1220, US Geological Survey, 1979.

[19] Brodeur, J. R., \& Nicaise, W. F., "Subsurface radionuclide assessment at Hanford using HPGe gamma-ray borehole geophysics," Transactions of the American Nuclear Society, 70(CONF-940602-), 1994.

[20] Saint-Gobain Ceramics \& Plastics, Inc., "NaI(Tl) and Polyscin $\mathrm{NaI}(\mathrm{Tl})$ Sodium Iodide Scintillation Material," 2020. [Online]. Available: sodium-iodide-material-data-sheet_0.pdf (saint-gobain.com). Accessed on: July 7, 2021

[21] McGregor, D. S., "Materials for gamma-ray spectrometers: Inorganic scintillators," Annual Review of Materials Research, 48, 245-277, 2018

[22] Quarati, F. G. A., et al., "Scintillation and detection characteristics of high-sensitivity $\mathrm{CeBr}_{3}$ gamma-ray spectrometers," Nuclear Instruments and Methods in Physics Research Section A: Accelerators, Spectrometers, Detectors and Associated Equipment 729: 596-604, 2013.

[23] Shah, K. S., Glodo, J., Higgins, W., Van Loef, E. V., Moses, W. W., Derenzo, S. E., \& Weber, M. J., " $\mathrm{CeBr}_{3}$ scintillators for gamma-ray spectroscopy," IEEE Transactions on Nuclear Science, 52(6), 31573159, 2005.

[24] Drozdowski, W., Dorenbos, P., Bos, A. J., Owens, A., \& Richaud, D., "Gamma radiation hardness of $\varnothing 1 " \times 1$ " $\mathrm{LaBr}_{3}$ : Ce, $\mathrm{LaCl}_{3}$ : Ce, and $\mathrm{CeBr}_{3}$ scintillators," In 2008 IEEE Nuclear Science Symposium Conference Record (pp. 2856-2858), IEEE, 2008.

[25] Kari Peräjärvi, S. T. U. K., Csome, F. C., \& Borg, J., "European Reference Network for Critical Infrastructure Protection: Novel detection technologies for nuclear security", 2018.

[26] John Caunt Scientific Ltd., "Gamma-Neutron Scintillation Detector," 2021. [Online]. Available: (CLYC)CLYC | JCS Nuclear Solutions (johncaunt.com). Accessed on: July 7, 2021.

[27] X-Z LAB, Inc., "GAGG(Ce) - Scintillation Crystal," 2021. [Online]. Available: https://www.X-zlab.com/product/gagg-scintillation-crystal/. Accessed on: July 7, 2021.

[28] Yoneyama, M., Kataoka, J., Arimoto, M., Masuda, T., Yoshino, M. Kamada, K., Usuki, Y., "Evaluation of GAGG:Ce scintillators for future space applications," Journal of Instrumentation, 13(02), P02023, 2018.

[29] Mesick, K. E., Bartlett, K. D., Coupland, D. D. S., Stonehill, L. C., "Effects of proton-induced radiation damage on CLYC and CLLBC performance," Nuclear Instruments and Methods in Physics Research Section A: Accelerators, Spectrometers, Detectors and Associated Equipment, 948, 162774, 2019.

[30] Hendriks, P. H. G. M., Limburg, J., De Meijer, R. J., "Full-spectrum analysis of natural $\gamma$-ray spectra," Journal of Environmental Radioactivity, 53(3), 365-380, 2001.

[31] Koomans, R., "Calibration of spectral gamma tools," The Medusa Institute, 2018. [Online]. Available: https://the.medusa.institute/display/GW/Calibration+of+spectral+gamma +tools. Accessed on: 15 February, 2021.

[32] IAEA, "X-ray and gamma-ray decay data: Standards for detector calibration and other applications," International Atomic Energy Agency (IAEA), 2005. [Online]. Available: https://wwwnds.iaea.org/xgamma_standards/. Accessed on: 7 July, 2021. 